\title{
Phenobarbitone, neonatal seizures, and video-EEG
}

\author{
G B Boylan, J M Rennie, R M Pressler, G Wilson, M Morton, C D Binnie
}

Arch Dis Child Fetal Neonatal Ed 2002;86:F165-F170

See end of article for authors' affiliations

Correspondence to: G B Boylan, NICU, 4th

Floor, Ruskin Wing, King's College Hospital, Denmark Hill, London SE5 9RS, UK; geraldine.boylan@kcl.ac.uk

Accepted for publication 8 December 2001

\begin{abstract}
Aims: To evaluate the effectiveness of phenobarbitone as an anticonvulsant in neonates.
Methods: An observational study using video-EEG telemetry. Video-EEG was obtained before treatment was started, for an hour after treatment was given, two hours after treatment was given, and again between 12 and 24 hours after treatment was given. Patients were recruited from all babies who required phenobarbitone (20-40 mg/kg intravenously over 20 minutes) for suspected clinical seizures and had EEG monitoring one hour before and up to 24 hours after the initial dose. An EEG seizure discharge was defined as a sudden repetitive stereotyped discharge lasting for at least 10 seconds. Neonatal status epilepticus was defined as continuous seizure activity for at least 30 minutes. Seizures were categorised as EEG seizure discharges only (electrographic), or as EEG seizure discharges with accompanying clinical manifestations (electroclinical). Surviving babies were assessed at one year using the Griffiths neurodevelopmental score.

Results: Fourteen babies were studied. Four responded to phenobarbitone; these had normal or moderately abnormal EEG background abnormalities and outcome was good. In the other 10 babies electrographic seizures increased after treatment, whereas electroclinical seizures reduced. Three babies were treated with second line anticonvulsants, of whom two responded. One of these had a normal neurodevelopmental score at one year, but the outcome for the remainder of the whole group was poor.

Conclusion: Phenobarbitone is often ineffective as a first line anticonvulsant in neonates with seizures in whom the background EEG is significantly abnormal.
\end{abstract}

$P$ henobarbitone remains the most frequently used first line treatment for neonatal seizures worldwide, in spite of accumulating evidence that it is ineffective in many babies. $^{1}$ Seizures in the newborn are associated with underlying conditions such as brain haemorrhage, stroke, meningitis, and hypoxic ischaemic encephalopathy. Many studies evaluating the effectiveness of phenobarbitone have evaluated only clinical control, as the electroencephalogram (EEG) was not recorded at the time of seizures. ${ }^{2-4}$ Recent studies have shown that seizures in the newborn are often clinically silent (or "electrographic"), and the extent of the electrographic seizure burden in the sick baby is often greatly underestimated. ${ }^{5-9}$ Electrographic seizures cannot be diagnosed without an EEG and even when clinical correlates are present they are often very subtle. Video-EEG has been shown to be the most useful technique available to identify, classify, and quantify neonatal seizures. ${ }^{59}$ Effective seizure control in the neonate, therefore, implies abolition of clinical and electrographic seizures.

EEG monitoring of treated neonatal seizures shows that most current treatments are often ineffective in suppressing abnormal electrical activity. ${ }^{17}$ Phenobarbitone is a potent sedative as well as a powerful anticonvulsant. Our previous work has shown that electrographic seizures were more common in babies who had been treated with phenobarbitone.' We, and others ${ }^{5}$ have observed that this drug sedates babies, suppressing clinical manifestations of seizures, but has little effect on the electrographic discharge.

It is still not known whether neonatal seizures, in particular, electrographic seizures are themselves harmful. However, evidence is accumulating regarding the potential detrimental effects of electroclinical and electrographic seizures in neonates. ${ }^{10-12}$ Animal studies suggest that seizures are deleterious to the development of the immature brain. ${ }^{13-16}$ Twenty to forty per cent of term neonates who suffer convulsions are subsequently handicapped, whereas in preterm neonates, adverse sequelae occur after seizures in $75-88 \%{ }^{17}$
The aim of this study was to use video-EEG to evaluate the effectiveness of phenobarbitone as a first line anticonvulsant in the neonate by careful examination of its effects on the duration of both electroclinical and electrographic neonatal seizures.

\section{PATIENTS AND METHODS \\ Patients}

From a prospective research study using video-EEG to monitor neonates at high risk of seizures, we included in this analysis only those neonates who fulfilled the following criteria:

(1) Electrographic or electroclinical seizures were present

(2) Monitoring started at least one hour prior to the administration of phenobarbitone

(3) Monitoring was continued for at least 1-2 hours immediately after treatment

(4) Monitoring was either continuous or repeated at regular intervals over subsequent days.

The ethics committee of King's College Hospital approved this study. Written informed parental consent was obtained from the parents of all babies studied.

\section{Therapy}

A loading dose of $20 \mathrm{mg} / \mathrm{kg}$ phenobarbitone was administered intravenously over a 15-20 minute period. Further loading doses were administered up to a maximum of $40 \mathrm{mg} / \mathrm{kg}$ if seizures proved difficult to control. Serum concentrations of phenobarbitone were measured using the CEDIA homogeneous enzyme immunoassay methodology (Roche Diagnostics, Lewes, UK) in most babies and as close as possible to the time of EEG monitoring. If seizures failed to respond or recurred after phenobarbitone therapy, clonazepam or phenytoin was used. 


\section{Table 1 Classification of EEG background activity \\ 1 Normal/mild Normal pattern for GA, including slightly abnormalities abnormal activity, e.g. mild asymmetries, mild voltage depression \\ 2 Moderate abnormalities \\ Discontinuous activity with $|\mathrm{B}|$ too long for GA. Clear asymmetry or asynchrony. Absence of age appropriate EEG features, e.g. delta brushes \\ 3 Major abnormalities Severe discontinuity in EEG for GA. Burst suppression pattern. No appropriate wake-sleep cycles for GA. Multi focal sharp waves or positive rolandic sharp waves \\ 4 Inactive EEG Background activity $<10 \mu \mathrm{V}$ \\ $G A$, gestational age; $|B|$, interburst intervals.}

\section{Video-EEG monitoring}

A Telefactor (Modac or Beehive) video-EEG system was used to record 12 channels of EEG using the 10-20 system of electrode placement modified for neonates $\left(\mathrm{F}_{4}-\mathrm{C}_{4}, \mathrm{C}_{4}-\mathrm{P}_{4}, \mathrm{P}_{4}-\mathrm{O}_{2}\right.$, $\left.\mathrm{F}_{3}-\mathrm{C}_{3}, \mathrm{C}_{3}-\mathrm{P}_{3}, \mathrm{P}_{3}-\mathrm{O}_{1}, \mathrm{~T}_{4}-\mathrm{O}_{2}, \mathrm{~T}_{3}-\mathrm{O}_{1}\right)$. A single channel electrooculogram was recorded from the upper outer canthus of the right eye to the lower outer canthus of the left eye and the sub-mentalis electromyogram was also recorded on one channel. The remaining two channels were configured to display ECG and respiration via an output from the baby's clinical monitor. A video recording was made of each baby for the entire duration of study. The EEG-polygraphy waveforms were embedded on to the video picture and recorded by a Panasonic video recorder on to conventional videotape.

Each baby was monitored as soon as possible after the first seizure. Recordings were continued until seizure control was obtained and then repeated within 24 hours unless the baby was too unstable to tolerate the initial handling required for EEG monitoring. If seizure control was not obtained the recording was continued until further treatment was administered.

\section{EEG analysis}

Each videotape was carefully reviewed and analysed for periods of (1) electroclinical seizure and (2) electrographic seizure. Each electroclinical and electrographic seizure was counted and timed. Results were then assigned to one of four time periods as follows:

- The hour immediately prior to treatment $\left(\mathrm{T}^{0}\right)$

- The first full hour after all phenobarbitone had been administered $\left(\mathrm{T}^{1}\right)$ (20 minutes was allowed for the loading dose of phenobarbitone to be administered)

- The second full hour after treatment was completed $\left(\mathrm{T}^{2}\right)$

- 12-24 hours after treatment $\left(\mathrm{T}^{24}\right)$.

The diagnosis of an electrographic seizure required the evolution of sudden, repetitive, evolving stereotyped forms with a definite beginning, middle, and end. ${ }^{18} \mathrm{~A}$ minimal ictal duration of 10 seconds was used for this study. Neonatal status epilepticus was defined as continuous seizure activity for at least 30 minutes or recurrent seizures for more than $50 \%$ of the entire recording duration. ${ }^{19}$ Seizure control was defined as complete elimination of all electrographic seizures. Background activity of the EEG was classified according to the criteria defined in table 1 . The main features of this classification have been defined previously. ${ }^{20}$

\section{Neurodevelopmental assessment}

All surviving babies had a neurodevelopmental assessment at 1 year using the Amiel-Tison Test, ${ }^{21}$ the Griffiths developmental scale for babies, ${ }^{22}$ and a neurological examination.
Neurodevelopmental outcome was classified as normal (Amiel-Tison score 0; Griffiths quotient $\geqslant 85 \%$ ) or abnormal (Amiel-Tison score 1 or 2; Griffiths quotient $<85 \%$ ) with minor or major sequelae (cerebral palsy, motor or sensory deficits, and/or epilepsy).

\section{Statistical analysis}

Wilcoxon tests for matched pairs tests were used to compare changes in total seizure duration before and after treatment in the pooled results from all babies. ${ }^{23}$ Seizures were divided into electrographic and electroclinical for all babies; seizure duration at time $\mathrm{T}^{0}$ was compared with seizure duration at time $\mathrm{T}^{1}$, and seizure duration at time $\mathrm{T}^{0}$ was also compared with $\mathrm{T}^{24}$.

\section{RESULTS}

\section{Subjects}

During a three year period, 33 babies had seizures but only 14 met the inclusion criteria for this study and had video-EEG monitoring at least one hour before a loading dose of phenobarbitone was administered.

Table 2 gives clinical information about the babies. Four term babies had hypoxic ischaemic encephalopathy. Three preterm babies had bilateral intracranial haemorrhage. Three babies had seizures caused by metabolic problems (hypoglycaemia in two and kernicterus in one). One baby had meningitis. Two preterm babies had evidence of birth depression and one otherwise healthy term baby had idiopathic seizures.

\section{EEG response to phenobarbitone}

Phenobarbitone concentrations were measured in 12 babies 24-48 hours after the first loading dose of phenobarbitone. One baby had a concentration of $46.9 \mathrm{mg} / \mathrm{l}$, which was above the therapeutic range (table 2, case 8 ). All other babies had concentrations within the therapeutic range. Immediately after phenobarbitone administration, all babies showed a transient attenuation of background activity lasting 10-20 minutes.

Table 3 shows the seizure duration in seconds for each baby in the hour before treatment, the hour after treatment, two hours after treatment, and 12-24 hours later. Three babies received a second loading dose of phenobarbitone the following day during EEG recording. The change in seizure duration following both doses is included for these babies.

Six babies were in neonatal status prior to anticonvulsant administration. Two babies had purely electrographic seizures before treatment, of which one was therapeutically paralysed. The remaining babies had electrographic and electroclinical seizures in the hour preceding treatment.

Seizure duration rather than the number of seizures per hour was used to determine the severity of seizure burden as the number of seizures alone could be misleading. This was particularly evident in babies with neonatal status who often had just one seizure in an hour but this seizure could last for up to an hour in some cases.

Four babies were treated with a second line anticonvulsant before the final EEG was performed because clinical seizure activity had reappeared. Wilcoxon tests for matched pairs revealed no significant change in total seizure duration in all babies when comparing before treatment, one hour after treatment, two hours after treatment, and the next day.

\section{Babies who responded to phenobarbitone therapy}

Four of the 14 babies responded to a loading dose of phenobarbitone. Two term babies had focal temporal lobe seizures with apnoea and tonic posturing (table 3, cases 1 and 2). The background EEG in both of these babies showed only mild abnormalities. One baby responded within an hour to a loading dose of phenobarbitone and remained seizure free. The other baby had approximately one seizure per hour prior to 
Table 2 Clinical details and outcome of all babies monitored

\begin{tabular}{ccccll}
\hline Case & GA & CA & BW $(\mathbf{g})$ & Aetiology & Outcome \\
\hline 1 & 41 & 41 & 4110 & Unknown & Normal \\
2 & 39 & 39 & 3590 & Hypoglycaemia & Normal \\
3 & 41 & 41 & 3410 & HIE & Mild \\
4 & 23 & 26 & 668 & Prematurity and BD & Moderate \\
5 & 40 & 40 & 2340 & Hypoglycaemia and unilateral ICH & Moderate \\
6 & 36 & 37 & 2580 & Kernicterus & Severe \\
7 & 28 & 34 & 908 & Meningitis & Severe \\
8 & 41 & 41 & 3712 & Bilateral ICH & Severe \\
9 & 30 & 31 & 1770 & Bilateral ICH & Died \\
10 & 25 & 25 & 700 & Prematurity, BD, myopathy & Died \\
11 & 34 & 35 & 2122 & HIE & Died \\
12 & 40 & 40 & 2624 & Rhesus disease and bilateral ICH & Died \\
13 & 34 & 35 & 1540 & HIE & Died \\
14 & 38 & 38 & 4114 & & \\
\hline
\end{tabular}

BW, body weight; GA, gestational age; CA, chronological age; HIE, hypoxic ischaemic encephalopathy; BD, birth depression; ICH, intracranial haemorrhage; IUGR, intrauterine growth retardation.

Table 3 Response of 14 babies to phenobarbitone administration during the EEG

\begin{tabular}{|c|c|c|c|c|c|c|c|c|c|}
\hline \multirow[b]{2}{*}{ Case } & \multirow[b]{2}{*}{ EEG grade } & \multicolumn{2}{|l|}{$\mathrm{T}^{0}$} & \multicolumn{2}{|l|}{$T^{1}$} & \multicolumn{2}{|l|}{$\mathrm{T}^{2}$} & \multicolumn{2}{|l|}{$\mathrm{T}^{24}$} \\
\hline & & $E$ & EC & E & EC & $E$ & EC & $\mathrm{E}$ & EC \\
\hline 1 & 2 & 0 & 158 & 0 & 0 & - & - & 0 & 166 \\
\hline 2 & 2 & 0 & NS & 0 & 0 & - & - & 0 & 0 \\
\hline 3 & 3 & 229 & 1885 & 0 & 0 & 133 & 1627 & $\ddagger^{2,3} \mathrm{O}$ & 0 \\
\hline 4 & 3 & 689 & 507 & 170 & 10 & 6 & 25 & 3600 & 0 \\
\hline 5 & 2 & 0 & 1376 & 0 & 506 & - & - & 0 & 669 \\
\hline 6 & 2 & 0 & 937 & 30 & 40 & - & - & 0 & 0 \\
\hline 7 & 4 & 0 & 3600 & 1066 & 346 & 23 & 50 & $\ddagger^{1} 0$ & 0 \\
\hline 8* & 3 & 0 & NS & 1869 & 0 & NS & 0 & NS & 0 \\
\hline $8 \dagger$ & 3 & NS & 0 & NS & 0 & - & - & $\ddagger^{2} 620$ & 0 \\
\hline 9* & 3 & 0 & NS & 0 & NS & 0 & NS & NS & 0 \\
\hline $9 \dagger$ & 3 & NS & 0 & NS & 0 & NS & 0 & 0 & 758 \\
\hline $10 *$ & 4 & 0 & 2580 & 1208 & 1193 & 2400 & 1200 & 3600 & 0 \\
\hline $10 \dagger$ & 4 & 3600 & 0 & 79 & 0 & 195 & 0 & 0 & 3600 \\
\hline 11 & 3 & 1280 & 0 & 120 & 0 & 0 & 0 & 0 & 0 \\
\hline 12 & 3 & 91 & 909 & 6 & 665 & - & - & $\ddagger^{3} 0$ & 1013 \\
\hline 13 & 3 & 0 & 639 & 613 & 579 & - & - & 856 & 0 \\
\hline 14 & 4 & 1457 & 0 & 0 & 0 & - & - & NS & 0 \\
\hline
\end{tabular}

$T^{0}$ refers to the hour immediately prior to treatment. $T^{1}$ refers to the hour after all phenobarbitone had been given. $T^{2}$ is the second hour after phenobarbitone had been given. $\mathrm{T}^{24}$ is one hour from the EEG recording the next day.

E, electrographic seizures; EC, electroclinical seizures; NS, neonatal status.

*First loading dose of phenobarbitone; †second loading dose of phenobarbitone; finfant also received a second line anticonvulsant before the recording at $\mathrm{T}^{24}$ (midazolam ${ }^{1}$, clonazepam ${ }^{2}$, and phenytoin ${ }^{3}$.

phenobarbitone. After phenobarbitone he had no further fits all night. He had one seizure during the EEG the next day but no further seizures were ever seen. Both of these babies were normal at follow up.

One baby with kernicterus caused by glucose 6-phosphate dehydrogenase deficiency, who had focal tonic seizures and a moderately abnormal background EEG, responded to phenobarbitone therapy within one hour (table 3, case 6). He had one further electroclinical seizure and one electrographic seizure after the loading dose of phenobarbitone. He continued seizure free on a maintenance dose of phenobarbitone. At follow up he has a moderate hearing impairment.

One preterm baby with birth depression associated with myopathy had focal short duration electrographic seizures. These were suppressed almost immediately by a loading dose of phenobarbitone and no further fits were ever seen (table 3, case 11). The background EEG showed severe abnormalities. This baby remained ventilator dependent and died three weeks later, after intensive care was withdrawn. Figure lA shows total seizure duration before treatment, one hour after treatment, and 12-24 hours later for these four babies.

Babies who did not respond to phenobarbitone therapy The remaining 10 babies did not respond to phenobarbitone therapy. All had moderate to severe background EEG abnormalities. Figure $1 \mathrm{~B}$ shows the change in total seizure duration before treatment, one hour after treatment, and 12-24 hours later in seven of these babies. Two babies did show a dramatic reduction in seizure burden one hour after treatment; however, by $\mathrm{T}^{24}$ seizures had reappeared. Four babies showed either continuation of clinical seizures or the re-emergence of clinical seizures before the final EEG was performed and were therefore treated with a second line anticonvulsant by $\mathrm{T}^{24}$ (see fig $\mathrm{lC}$ ).

\section{Response to a second loading dose of phenobarbitone}

At the time of this study it was unit policy to treat only those seizures with accompanying clinical manifestations. However, if electrographic seizures persisted for over 24 hours a second load of phenobarbitone was given, particularly if these electrographic seizures represented neonatal status. Three babies had two separate loading doses of phenobarbitone given during EEG monitoring. In one baby (table 3, case 8 ) the initial effect of phenobarbitone was to temporarily reduce the seizure burden. This baby was in electroclinical status epilepticus in the hour before phenobarbitone was given. Clinical signs were very subtle and difficult to assess, consisting of intermittent eye blinking and slight limb stiffening. After phenobarbitone, the seizure burden reduced to 1869 seconds 

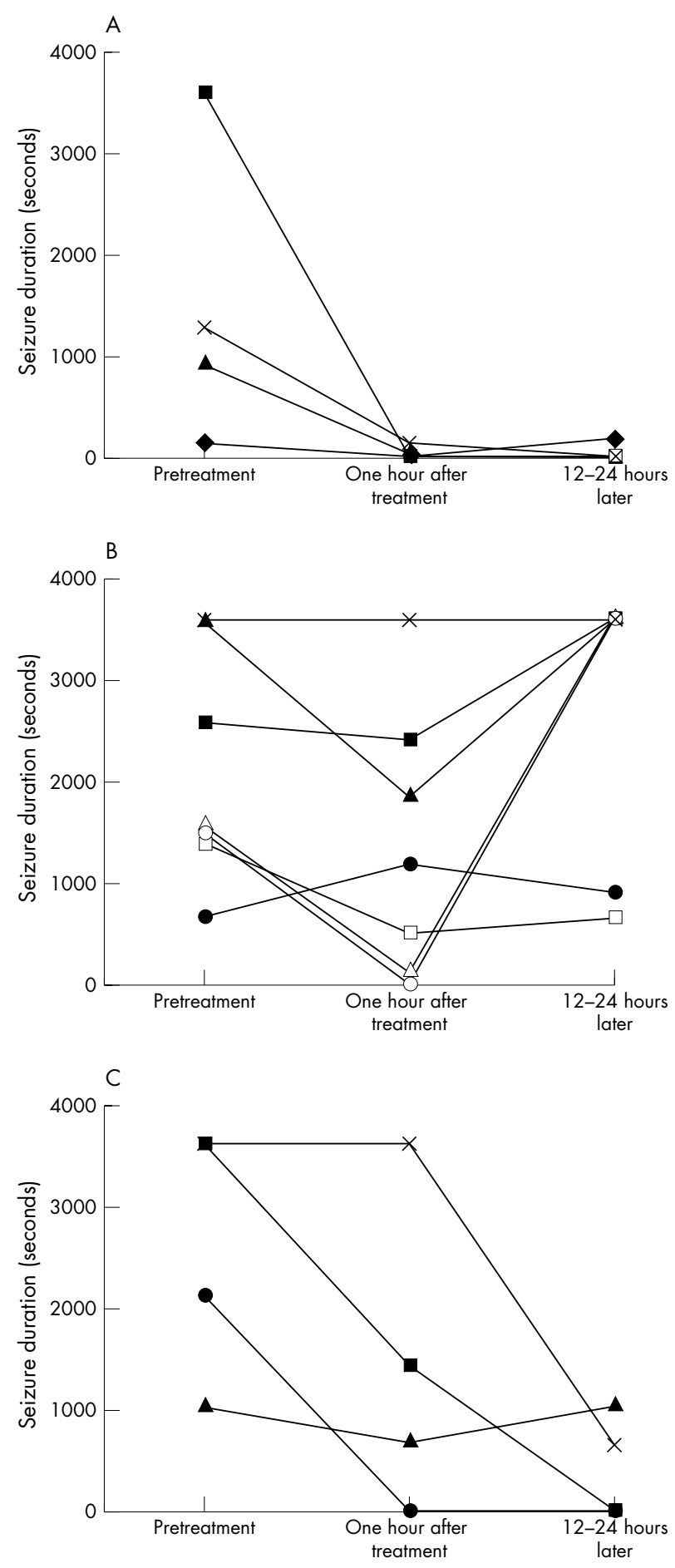

Figure 1 Change in total seizure duration for $(A)$ four babies who responded to phenobarbitone therapy, (B) seven of ten babies who did not respond to phenobarbitone therapy, and (C) four babies who did not respond to phenobarbitone therapy but received a second line anticonvulsant before the final EEG at $\mathrm{T}^{24}$. One baby appears twice (in B and C) as she did not respond to two loading doses of phenobarbitone and later received a second line anticonvulsant before the final EEG (case 8, table 3).

in the first hour. Clinically, there appeared to be a dramatic response as these 1869 seconds were pure electrographic seizures. While technically this is still neonatal status (more than $50 \%$ of the recording time), it did signify some improvement. By the second hour after treatment, however, the baby had returned to electrographic status for almost $100 \%$ of the recording time. Clinically the baby was still thought to be improving as clinical signs of seizures were no longer obvious. In fact, only the clinical manifestation of the seizures had been removed. The second loading dose of phenobarbitone was given after 24 hours but failed to reduce electrographic seizures; seizures could only be reduced 12 hours later by the addition of clonazepam.

A second baby with bilateral intraventricular haemorrhage also had two loading doses of phenobarbitone during the EEG (table 3, case 9 ). This baby was in neonatal status and conversion of electroclinical seizures to electrographic seizures was also seen. The first load of phenobarbitone did not have any effect on seizure burden for two hours. After this time seizures became purely electrographic. The EEG the next day, 12 hours later, showed a reduced seizure burden but the re-emergence of electroclinical seizures. At this point a second dose of phenobarbitone was given but failed to have any further effect on seizure burden. Finally, the third baby who received two doses of phenobarbitone failed to respond. Electroclinical seizures were converted to electrographic seizures following the first dose of phenobarbitone and the second dose of phenobarbitone did not have any further effect (table 3, case 10). In these three babies it was concluded that the addition of a second load of phenobarbitone to control seizures that had failed to respond to the initial load did not produce any further benefits.

\section{Electroclinical dissociation}

Measuring total seizure duration alone does not fully explain the apparent clinical reduction in seizure burden seen after treatment in this study. Total electrographic and electroclinical seizure duration were measured separately in each baby. Electroclinical seizures were more common before treatment. One hour after treatment, electroclinical seizures reduced significantly $(\mathrm{p}=0.001$, median change -158 seconds, 95\% CI: -1047 to 0$)$. By time $\mathrm{T}^{24}$ electroclinical seizures were still significantly reduced $(\mathrm{p}=0.030$, median change $-573,95 \%$ CI: -3098 to 0 ). There was a trend for electrographic seizures to increase in association with electroclinical seizure reduction, but the change was not significant. Figure 2 shows the results from this analysis in seven of the 10 babies who did not respond to phenobarbitone treatment and who did not receive a second line anticonvulsant. It is clear from individual results, however, that in most babies electroclinical seizures reduced and electrographic seizures increased after phenobarbitone. In other words, following phenobarbitone administration, the clinical component of the seizures reduced while electrographic seizures continued (electroclinical dissociation).

\section{Neurodevelopmental outcome}

At follow up, in this group of babies with electrographic and/or electroclinical seizures, five had died, three had severe neurological impairment, four had mild to moderate impairment, and two were normal. All babies with a severely abnormal EEG (severely depressed trace or neonatal status), died, or had severe neurological impairment (table 2). Most babies who did not respond to phenobarbitone alone either died or had neurological abnormalities.

\section{DISCUSSION}

Using careful analysis of video-EEG we have shown that in the majority of neonates phenobarbitone administration is followed by a decrease in clinical manifestations of seizures while electrographic seizures continue. Phenobarbitone was only effective in $29 \%$ of babies. In our experience, these were babies with normal background EEGs or mild to moderate background abnormalities and relatively low seizure burden. Response to a loading dose of $20 \mathrm{mg} / \mathrm{kg}$ phenobarbitone was very rapid in this group, who had a good prognosis. Three of the four babies who responded were term and had metabolic 
Case 4

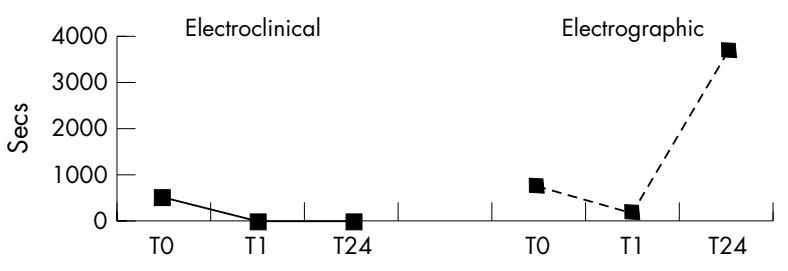

Case 8

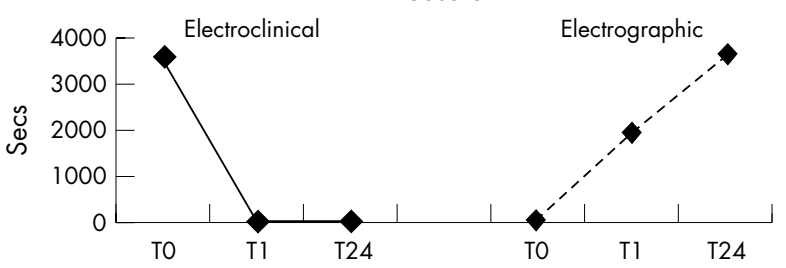

Case 10

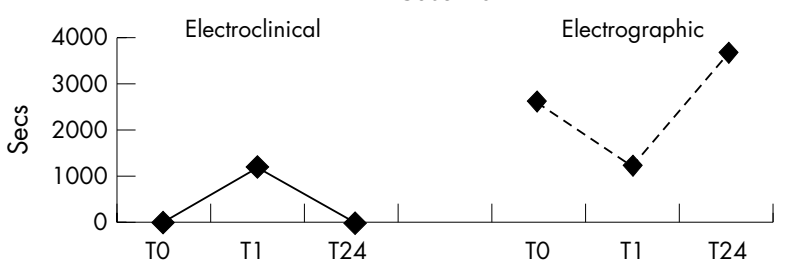

Case 14

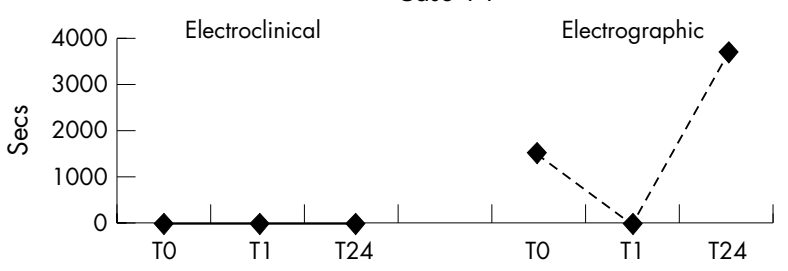

Case 5

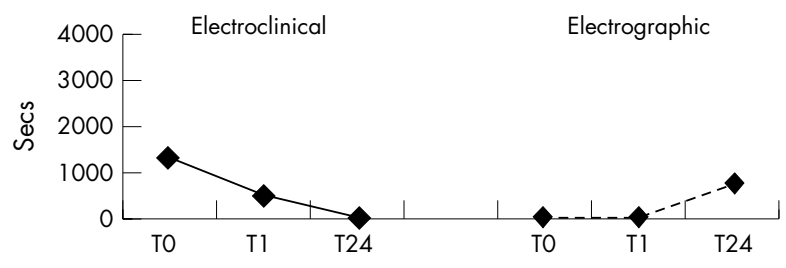

Case 9

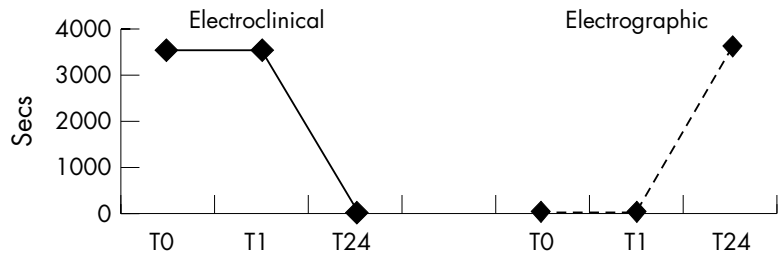

Case 13

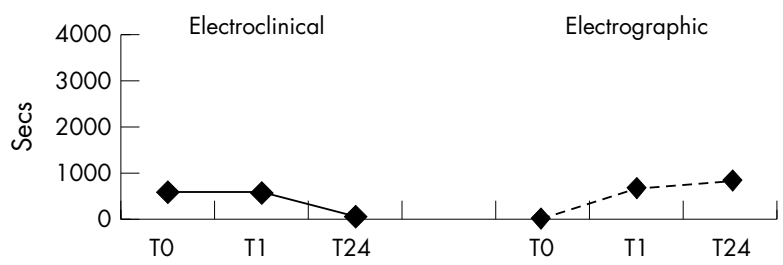

Figure 2 Change in electroclinical and electrographic seizure duration in seven of 10 babies who did not respond to phenobarbitone therapy.

abnormalities or seizures of unknown cause. The fourth responder was a preterm baby with birth depression and myopathy. He had short duration occipital electrographic seizures.

Babies who did not respond to phenobarbitone had abnormal background EEGs and over half were in neonatal status prior to the commencement of therapy. Three babies were given a further loading dose of phenobarbitone during EEG monitoring after the first dose failed to control seizures. Gal and colleagues ${ }^{4}$ achieved clinical control in up to two thirds of babies given a loading dose of $40 \mathrm{mg} / \mathrm{kg}$. The EEG was not monitored so it was not possible to assess whether phenobarbitone merely reduced the clinical component of the seizures. No further reduction in electrographic seizures was seen following the additional dose of phenobarbitone given to three babies in our EEG study. Four of the 10 babies who did not respond to phenobarbitone therapy were treated with a second line anticonvulsant; seizures were abolished in a further two, one of whom had a normal neurodevelopmental outcome.

Phenobarbitone has been used as first line treatment for neonatal seizures worldwide for over 30 years. Despite the fact that babies with seizures have a poor outcome, ${ }^{912}$ there have been no formal trials to develop a more effective treatment strategy for seizure control. Until recently, electrographic seizures were not considered harmful to the developing brain, and there was no pressure to treat to electrical quiescence. ${ }^{12}$ If the clinical manifestations accompanying a seizure discharge diminished or disappeared following anticonvulsant administration, the treatment was deemed a success. Our results show that electrographic seizures continue in this situation. Of course, babies cannot communicate in the same way as adults, meaning that aura and sensory seizure manifestations cannot be measured at all. In babies who show electroclinical dissociation after phenobarbitone treatment, all we can say is that the previous clinical correlates were abolished.

This study shows the importance of EEG monitoring as a tool with which to measure seizure control in the neonate. The frequency with which the neonate shows electroclinical dissociation makes monitoring of treatment using clinical measures alone difficult to justify, given the accumulating evidence that electrographic seizures are equally important and have adverse effects on the developing brain.

The number of babies studied was small because we could only monitor those babies in whom it was possible to attach electrodes and eliminate interference quickly and where it was possible to record for one hour prior to therapy. These babies were recruited from a group of high risk babies; in many, seizures were not suspected at the time of monitoring or clinical seizure activity was subtle. In some babies there was doubt about the nature of abnormal movements so treatment was not commenced until the EEG confirmed that the abnormal movements were seizures.

To our knowledge this is the first study to use video-EEG to quantify the change in both electroclinical and electrographic seizure before and after phenobarbitone treatment in a group 
of neonates. We conclude from our results that phenobarbitone is ineffective as a first line anticonvulsant treatment in babies with severe seizures in whom the background EEG is also severely abnormal. In these babies, the addition of a second dose of phenobarbitone up to the maximum dose of 40 $\mathrm{mg} / \mathrm{kg}$ does not appear to have any additional benefits. In our view, babies who fail to respond to phenobarbitone within two hours should be treated with a second line anticonvulsant. Painter and colleagues ${ }^{1}$ have shown that phenytoin is equally ineffective in controlling seizures as phenobarbitone. We agree wholeheartedly with these workers that there is an urgent need to develop a safe and effective treatment strategy for neonatal seizures.

\section{ACKNOWLEDGEMENTS}

G Boylan and G Wilson are supported by the National Lotteries Charities Board administered by the Fund for Epilepsy (grant RB214431). This project has also been supported by an equipment grant from the Bernard Sunley Foundation. We are very grateful for the continued support of nursing and medical staff at the NICU, King's College Hospital, and we would like to thank all parents who gave permission for us to study their babies. Without them, progress would be impossible.

\section{Authors' affiliations}

G B Boylan, J M Rennie, G Wilson, M Morton, Neonatal Intensive Care Unit, King's College Hospital, Denmark Hill, London SE5 9RS, UK R M Pressler, C D Binnie, Department of Clinical Neurophysiology, King's College Hospital

\section{REFERENCES}

1 Painter MJ, Scher MS, Stein AD, et al. Phenobarbital compared with phenytoin for the treatment of neonatal seizures. N Engl J Med 1999;341:485-9.

2 Gilman JT, Gal P, Duchowny MS, et al. Rapid sequential phenobarbital treatment of neonatal seizures. Pediatrics 1989:83:674-8.

3 Van Orman CB, Darwish HZ. Efficacy of phenobarbital in neonatal seizures. Can J Neurol Sci 1985:12:95-9.

4 Gal P, Toback J, Boer HR, et al. Efficacy of phenobarbital monotherapy in treatment of neonatal seizures - relationship to blood levels. Neurology 1982;32:1401-4.
5 Mizrahi EM, Kellaway P. Characterization and classification of neonatal seizures. Neurology 1987;37:1837-44.

6 Legido A, Clancy RR, Berman PH. Recent advances in the diagnosis, treatment, and prognosis of neonatal seizures. Pediatr Neurol 1988:4:79-86.

7 Connell J, Oozeer R, de Vries L, et al. Continuous EEG monitoring of neonatal seizures: diagnostic and prognostic considerations. Arch Dis Child 1989;64:452-8.

8 Scher MS, Painter M, Bergman I, et al. EEG diagnosis of neonatal seizures: clinical correlations and outcome. Pediatr Neurol 1989;5:17-24

9 Boylan GB, Pressler RM, Rennie JM, et al. Outcome of electroclinical, electrographic and clinical seizures in the newborn infant. Dev Med Child Neurol 1999:41:819-25.

10 Borch K, Pryds O, Holm S, et al. Regional cerebral blood flow during seizures in neonates. J Pediatr 1998;132(3 Pt 1):431-5.

11 Boylan GB, Panerai RB, Rennie JM, et al. Cerebral blood flow velocity during neonatal seizures. Arch Dis Child Fetal Neonatal Ed 1999:80:F105-10

12 McBride MC, Laroia N, Guillet R. Electrographic seizures in neonates correlate with poor neurodevelopmental outcome. Neurology 2000;55:506-13.

13 Wasterlain CG. Recurrent seizures in the developing brain are harmful. Epilepsia 1997;38:728-34.

14 Holmes GL, Gairsa JL, Chevassus-Au-Louis N, Ben Ari Y. Consequences of neonatal seizures in the rat: morphological and behavioural effects. Ann Neurol 1998:44:845-57.

15 Huang L, Cilio MR, Silveira DC, et al. Long-term effects of neonatal seizures: a behavioural, electrophysiological, and histological study. Brain Res Dev Brain Res 1999;1 18:99-107.

16 Villeneuve N, Ben Ari Y, Holmes GL, Gaiarsa JL. Neonatal seizures induced persistent changes in intrinsic properties of CAl rat hippocampal cells. Ann Neurol 2000;47:729-38

17 Scher MS, Aso K, Beggarly ME, et al. Electrographic seizures in preterm and full-term neonates: clinical correlates, associated brain lesions, and risk for neurologic sequelae. Pediatrics 1993;91:128-34.

18 Clancy RR, Legido A. The exact ictal and interictal duration of electroencephalographic neonatal seizures. Epilepsia 1987;28:537-41.

19 Scher MS, Hamid MY, Steppe DA, et al. Ictal and interictal electrographic seizure durations in preterm and term neonates. Epilepsia 1993;34:284-8.

20 Pressler RM, Boylan GB, Morton M, et al. Early serial EEG in hypoxic ischaemic encephalopathy. Clin Neurophysiol 2001;112:31-7.

21 Amiel-Tison CGA. Neurological assessment during the first year of life. Oxford: Oxford University Press, 1986

22 Griffiths R. The abilities of babies, London: ARIC, 1976

23 Stata statistical software. College Station, TT: Stata Corporation, 1997. 